\title{
Genome-wide scans of selection highlight the impact of biotic and abiotic constraints in natural populations of the model grass Brachypodium distachyon.
}

\author{
Yann Bourgeois ${ }^{1}$, Christoph Stritt ${ }^{2}$, Jean-Claude Walser ${ }^{3}$, Sean P. Gordon ${ }^{4}$, John P.
} Vogel $^{4}$, Anne C. Roulin ${ }^{2 *}$

7

8

9

10

${ }^{1}$ New York University Abu Dhabi, PO Box 129188, Saadiyat Island, Abu Dhabi,

11 United Arab Emirates

$12{ }^{2}$ Institute of Plant and Microbial Biology, University of Zürich, Zollikerstrasse 107,

138008 Zürich, Switzerland

$14{ }^{3}$ Genetic Diversity Centre, ETH Zürich, Universitätstrasse 16, Zurich, Switzerland

$15{ }^{4}$ DOE Joint Genome Institute, Walnut Creek, CA 94598, USA.

16

$17 *$ Corresponding author: anne.roulin@botinst.uzh.ch

18

19 Key words: local adaptation, genome scans of selection, demographic history, 20 grasses, Brachypodium distachyon, host-pathogen interaction, machine learning. 


\section{Summary}

23 Grasses are essential plants for ecosystem functioning. Quantifying the selective

24 pressures that act on natural variation in grass species is therefore essential regarding

25 biodiversity maintenance. In this study, we investigate the selection pressures that act

26 on two distinct populations of the grass model Brachypodium distachyon without

27 prior knowledge about the traits under selection. We took advantage of whole-

28 genome sequencing data produced for 44 natural accessions of $B$. distachyon and used

29 complementary genome-wide scans of selection (GWSS) methods to detect genomic

30 regions under balancing and positive selection. We show that selection is shaping

31 genetic diversity at multiple temporal and spatial scales in this species and affects

32 different genomic regions across the two populations. Gene Ontology annotation of

33 candidate genes reveals that pathogens may constitute important factors of positive

34 and balancing selection in Brachypodium distachyon. We eventually cross-validated

35 our results with QTL data available for leaf-rust resistance in this species and

36 demonstrate that, when paired with classical trait mapping, GWSS can help

37 pinpointing candidate genes for further molecular validation. Thanks to a near-base

38 perfect reference genome and the large collection of freely available natural

39 accessions collected across its natural range, $B$. distachyon appears as a prime system

40 for studies in ecology, population genomics and evolutionary biology. 


\section{Introduction}

44 Grasses cover more than $40 \%$ of the world land area (Gibson, 2009) and dominate a 45 wide variety of ecosystems, from tropical to temperate regions (Clayton, 1981; 46 Gibson, 2009). Grasses also play a key role in eco- and agrosystem functioning as 47 they provide habitats for many animal species (Groves, 2000) and represent the main 48 source of grain and forage (Stromberg 2011). Increasing crop production to meet the food and energy requirements of the world's growing population is, however, putting great pressure on natural grasslands (Wallace, 1997; Helm et al., 2009; Ceballos et al., 2010). Faced with constant deterioration and fragmentation due to human activities (Kiviniemi, 2002), these ecosystems are highly endangered (Ceballos et al., 2010), but little is known about their evolutionary resilience. Assessing the genetic basis of adaptation and quantifying the selection pressures that act on natural variation in grass species is therefore crucial with respect to biodiversity maintenance and food security.

To date, reciprocal transplant experiments have been extensively used to test for adaptive differentiation across populations (for review see Savolainen et al., 2013). Based on a "home vs. foreign" effect on fitness, reciprocal transplants are indeed powerful to unravel overall genotype by environment $(\mathrm{GxE})$ interactions and demonstrated the prevalence of local adaptation in grasses and plants in general (for review see Bischoff et al., 2006; Wadgymar et al., 2017). However, reciprocal transplant experiments use information such as survival, vegetative growth or seed production to measure the effect of the habitat on fitness (Bischoff et al. 2006). Hence they provide little insight into the functional and genetic bases of adaptation, unless combined with trait mapping such as quantitative trait locus (QTL) analyses and genome-wide association studies (GWAS) (Latta 2009). QTL analyses and GWAS, on the other hand, are largely constrained by the effort and time required for highresolution mapping. In grasses, while these trait-by-trait approaches have been valuable to decipher the genetic architecture of important characters with regard to crop genetic improvement (Huang et al. 2002; Barbieri et al. 2012; Morris et al. 2013; Slavov et al. 2014), they remain of limited value to grasp the overall selective forces that act on natural populations.

An efficient alternative to provide insights about evolutionary forces in natural 
76 then describing their function and the type of selection acting on them (Mitchell-Olds et al. 2007). For instance, new mutations that are beneficial in some populations can be positively selected and are more likely to quickly increase in frequency. Such socalled selective sweeps tend to reduce genetic diversity, increase differentiation among populations, and lead to extended haplotypes in the vicinity of the locus under selection due to genetic hitchhiking (Nielsen 2005; Hermisson 2009). Various genome-wide selection scans (GWSS) methods have been developed to detect such footprints of positive selection (Tang et al. 2007; Gautier et al. 2012; Stamatakis et al. 2013; Messer 2015) and GWSS are now emerging as complementary approaches to classical trait mapping.

A first class of GWSS is based on cross-population comparison. These methods scan the genome by comparing the lengths of haplotypes across populations and are powerful to identify hard selective sweeps (Tang et al., 2007), i.e. regions in the genome where one haplotype has undergone recent and near-complete fixation in one of the studied populations in response to selection (Figure 1A). Yet, if the advantageous mutation has been present in the population for long enough and recombined before selection took place, then several different haplotypes may increase in frequency (Figure 1B). Such events ("soft sweeps"), as well as partial hard sweeps, are less efficiently detected by GWSS based on cross-population comparison (Schlamp et al. 2016) since they do not erase polymorphism to the same extent as completed hard sweeps. Soft-sweeps can be yet detected by a second class of GWSS which are also based on extended haplotypes but are applied at the within population level. These scans, by measuring mean pairwise differences in haplotype length along the genome in a given population, can indeed identify loci under selection independently of the number of haplotypes (Figure 1B) or of the completeness of the sweep (Schlamp et al. 2016). Therefore, within-population GWSS can detect not only soft-sweeps, but also mutations that are undergoing selection and did not reach complete fixation within a population.

Scans for positive selection are nonetheless not exempt of shortcomings and, as any computational approach, can lead to the detection of false positives. First, these tests often rely on the assumption that regions above a given threshold for the test statistics should be enriched in selected genes. However, defining which threshold should limit the amount of false positives is not straightforward (Pavlidis et al. 2012). In addition, 
109 demographic events such as bottleneck may also lead to the fixation of long 110 haplotypes and mimics signal of selection (Schrider \& Kern, 2016). This is especially

111 true when the bottleneck is recent and that recombination did not have enough time to

112 break haplotype blocks. While some GWSS are now able to incorporate bottlenecks

113 or expansions in their models (Schrider \& Kern, 2016), disentangling the effects of

114 selection from demography remains notoriously challenging as it implies to precisely

115 model population size evolution over time. Comparing and validating cross- and

116 within-population GWSS with approaches that take into account the demographic

117 history of each population may therefore constitute the best way to identify genes

118 under different regimes of positive selection with high confidence.

119 Furthermore, while local adaptation is commonly associated to positive selection on 120 new advantageous polymorphisms, recent studies have demonstrated that balancing 121 selection is also playing an important role in this evolutionary process (Mitchell-Olds 122 et al. 2007; Rasmussen et al. 2014; Wu et al. 2017). The term balancing selection is 123 an "umbrella" concept (Fijarczyk \& Babik, 2015) which describes the maintenance of 124 genetic diversity over longer periods of time through adaptation to spatial 125 heterogeneity, heterozygote advantage and negative frequency-dependent selection 126 (Mitchell-Olds et al. 2007; Rasmussen et al. 2014). This process is more difficult to 127 detect than positive selection (Fijarczyk \& Babik, 2015) since older alleles had more 128 time to recombine and may lead to narrow signatures around selected sites (Teixeira 129 et al. 2014). As a consequence, the effect of balancing selection is still largely 130 overlooked in genome scans, which remain strongly biased towards the detection of 131 recent positive selection (Hassl \& Payseur, 2016). It is now possible, however, to 132 model the coalescent process along chromosomes and to identify genomic regions 133 with unusually long times to the most recent common ancestor (TMRCA, Figure 1C), 134 a signature which can arise through balancing selection (Rasmussen et al. 2014).

135 In this study, we capitalize on the near base-perfect quality of the reference genome of 136 the Mediterranean grass Brachypodium distachyon (https://phytozome.jgi.doe.gov) to 137 investigate how both positive and balancing selection are shaping diversity in this 138 species. In the last decade, $B$. distachyon has been developed as a powerful model for 139 research on temperate grass species as it is closely related to major crop cereals and to 140 some of the grasses used for biofuel production (The International Brachypodium 141 Consortium 2010). Entirely sequenced, its small diploid genome (272Mb) is fully 
142 assembled into five chromosomes and has been exhaustively annotated (The

143 International Brachypodium Consortium 2010). In addition, B. distachyon is broadly

144 distributed around the Mediterranean rim (Dell'Acqua et al., 2014; Gordon et al.,

145 2014; Tyler et al., 2016), providing access to natural populations from contrasting

146 habitats for which a large collection has been collected. It constitutes therefore a

147 unique and prime system to investigate the genetic basis of local adaptation in natural

148 grass populations, opening the way to further fundamental and applied research.

149 We took advantage of whole-genome sequencing data produced for 44 B. distachyon 150 natural accessions originating mainly from Spain and Turkey (Gordon et al., 2017).

151 Those accessions are genetically distinct, occur in the eastern and western outer range

152 of the species distribution and were selected to maximize genetic differentiation and

153 identify regional selective pressures that may have been important during

154 recolonization following the last glacial maximum (Stritt et al. 2018). Making use of

155 the 6 million SNPs we identified in these genomes, we combined a cross-population

156 (Rsb; Tang et al., 2007) and a within-population scan (H-scan; Schlamp et al., 2016)

157 to an integrated demography approach (diploS/HIC; Kern \& Schrider, 2018) to detect

158 high-confidence genomic regions under different regimes of positive selection. We

159 also tested for regions under balancing selection with the program ARGweaver

160 (Rasmussen et al. 2014) and asked: i) At what time and geographical scale is selection

161 acting in B. distachyon populations? ii) What are the selective constrains that shape

162 diversity and adaptation in these populations? iii) Can we recover candidates for local

163 adaptation identified from QTL analyses performed in controlled conditions?

164

\section{Results}

166 Population structure and genetic diversity

167 In this study, we used whole-genome sequencing data (paired-end; Illumina 168 technology) with a 86-fold median coverage of $44 \mathrm{~B}$. distachyion accessions 169 originating from Turkey, Iraq, Spain and France (Figure 2A, Table S1, Gordon et al., 170 2017). After filtering, we identified 6,204,029 SNPs. An Admixture analysis, where $171 \mathrm{~K}=2$ was identified as the best model (Figure S1), highlighted two distinct genetic 172 clusters, an eastern and a western one, with extremely little admixture between the 173 two (Figure 2B). For the rest of the study, and even though these accessions belong to 
174 two genetic groups rather than stricto sensu populations, accessions from Turkey and

175 Iraq will be referred to as the eastern population while accessions from Spain and

176 France will be referred to as the western population.

177 The western population showed a lower level of nucleotide diversity (median $\pi$ value

178 in east $=0.0021$, median $\pi$ value in west $=0.00186$; Wilcoxon test; $\mathrm{P}$-value $<2.2 \mathrm{e}-16$,

179 Figure 2C) than the eastern one. Excluding the reference accession Bd21, which has

180 been artificially inbred before sequencing, the level of heterozygosity in these

181 accessions ranges from 4 to $17.4 \%$ (Table S1) but median values (7.8 and 6.3 in the

182 eastern and western population respectively) did not statistically differ between the

183 two populations (Wilcoxon test; P-value $=0.31$ ). Eventually, linkage disequilibrium

184 (LD) decays faster in the western population than in the eastern one (Figure $2 \mathrm{D}$ ).

185

186 Functional clustering of the genome of B. distachyon and subsequent filtering

187 To assess whether the genome of $B$. distachyon harbors functional clusters of genes,

188 we first performed a GO annotation for the 32,712 genes annotated in the reference

189 genome. We then controlled for potential gene clustering by following the procedure

190 described in (Al-Shahrour et al., 2010). Briefly, we split the genome into overlapping

191 windows of 50 consecutive genes and performed enrichment analyses on each

192 window. We identified 272 windows significantly enriched for at least one biological

193 process (Table S2). Several windows were enriched for processes that may be

194 associated to adaptation to local environmental conditions such response to stress and

195 defense response (Table S2).

196 A classical approach applied to analyze the results of GWSS consists in selecting

197 genomic regions containing top $1 \%$ outliers for signals of selection and then assessing

198 whether some biological functions or processes are significantly over-represented in

199 these regions through a Gene Ontology (GO) annotation. We anticipated that the non-

200 random organization of $B$. distachyon genome we observed could lead to an over-

201 representation of some biological pathways or functions in small genomic regions and

202 to an artificial enrichment for some GO terms in GWSS 1\% outlier regions (Pavlidis

203 et al. 2012). This prompted us to narrow down the top 1\% outlier regions by keeping

204 only the genes located at and in the immediate vicinity ( $-10 \%$ of the peak value) of

205 each of the peaks of selection for the cross- (Rsb test) and within-(H-scan) population

206 scans of selection presented in the following section. In contrast, the outputs of the 
207 coalescence and demography approaches, which are both window-based (see 208 methods), were not filtered.

209

210 Detection of hard selective sweeps through cross-population contrasts

211 When new mutations providing a selective advantage rise in frequency through

212 positive selection, neutral mutations that are physically close tend to remain strongly

213 linked to them. Recent positive selection should therefore lead to a signature of long

214 haplotypes near selected mutations. We used the Rsb test (see methods) to contrast

215 the two populations (Tang et al. 2007) and to detect regions where one haplotype

216 (hard sweep, Figure 1A) reached near or complete fixation a recent past in one of the 217 two populations. By doing so, we identified 312 regions harboring 824 genes and 319

218 regions harboring 1212 genes potentially under selection in the eastern and western 219 populations respectively.

221 Detection of soft or ongoing selective sweeps through a within-population scan

222 The Rsb test is less efficient to detect soft-sweeps, i.e. regions where more than one 223 haplotype increased in frequency (Figure 1B), or to identify ongoing selective sweep 224 where not all individuals within the population display haplotype extension in the 225 region under selection. As an alternative, we used the program $\mathrm{H}$-scan (see methods) 226 to detect loci associated to soft selective sweeps or undergoing positive selection 227 within each population. We identified 142 regions harboring 487 genes and 79 228 regions harboring 463 genes in the eastern and western population respectively. As 229 expected given the specificity of each test, only 49 and 58 genes were common to the $230 \mathrm{H}$-scan and Rsb approaches in the eastern and the western populations respectively.

232 Integration of demography and detection of high-confidence gene sets under positive 233 selection

234 As presented in the introduction, bottlenecks can mimic signals of selection as they 235 also lead to the fixation of long haplotypes. To alleviate this issue, we applied 236 diploS/HIC, a recently developed machine-learning approach to detect selective 237 sweeps and classify them as hard or soft (Schrider and Kern 2016; Kern and Schrider 238 2018) while integrating the demographic history of the eastern and western population 
239 previously inferred by (Stritt et al. 2018). One of the major advantages of diploS/HIC

240 is that the algorithm is robust to demographic model misspecification (Schrider and

241 Kern 2018). By providing a confusion matrix, it also estimates false positive and false

242 negative rates and allows a better assessment of the confidence levels expected under

243 different scenarios of selection.

244 We first trained the program to identify regions under positive selection. To do so, 245 genomic windows were simulated under neutrality, with a hard sweep or with a soft 246 sweep (see methods). The algorithm was able to recover from the simulations $71 \%$ 247 and $91.5 \%$ of hard sweeps in western and eastern populations respectively (Figure S2 248 for and example of confusion matrix). For soft sweeps, these proportions were of $71 \%$ 249 and $83.7 \%$. The proportion of neutral simulations being classified as hard sweeps was 250 low, accounting for $1.25 \%$ and $0.04 \%$ of the simulations in western and eastern 251 populations. These proportions were higher for soft sweeps, particularly in the 252 western population $(11.28 \%)$ but still remained low in the eastern population $(2.6 \%)$.

253 These results suggest a good sensitivity and specificity of diploS/HIC to detect 254 regions that are under selection, especially in the eastern population.

255 We then ran diploS/HIC on our actual dataset. Windows classified as selective sweeps 256 by diploS/HIC on this dataset recovered 34\% (164 genes) and 38\% (312 genes) of the 257 genes detected by the H-scan and Rsb tests in the eastern population, as well as 55\% 258 (258 genes) and 53\% (644 genes) of the genes detected by the H-scan and Rsb tests in 259 the western population. DiploS/HIC recovered more genes detected by the Rsb and H260 scan tests than expected by chance (Fisher test, all P-values $<1.8 \mathrm{e}-12$ ). These four 261 new genes sets (intersects between diploS/HIC-Rsb East, diploS/HIC-Rsb West, 262 diploS/HIC-Hscan East, diploS/HIC-Hscan West) are considered as high-confidence 263 genes under positive selection (Table S3 for a list). Unless mentioned otherwise, those 264 sets will be referred to as genes under positive selection in the discussion.

265 The high-confidence gene set diploS/HIC-Rsb of the eastern population displayed a 266 significant enrichment for genes involved in stress response and defense response 267 (Table 1). Among other GO term enrichments, the eastern high-confidence gene set 268 diploS/HIC-Hscan was significantly enriched for gene involved in response to 269 cadmium ion (see Table 1 for more significant GO terms). As we observed that some 270 of the well-known types of plant resistance genes (i.e. R-genes harboring NBS-LRR 271 domains) were not associated with the process of defense response in the GO 
272 analysis, we specifically counted how many genes containing NBS-LRR domains

273 were present in the four high-confidence gene sets and compared those to the number

274 genes with NBS-LRR domains annotated in the whole genome. Both high-confidence

275 gene sets (diploS/HIC-Hscan and diploS/HIC-Rsb) of the eastern population

276 displayed a significant enrichment for R-genes ( $\mathrm{P}$-value $=1.7 \mathrm{e}-06$ and $1.3 \mathrm{e}-05$

277 respectively). This was not the case for the high-confidence gene sets of the western

278 population (both P-value > 0.6).

279

280 Genomic regions affected by positive selection

281 The regions common to diploS/HIC-Hscan contained slightly more genes in the 282 western than in the eastern population (Wilcoxon test, P-value=0.04; Figure 3A), 283 while there was no significant difference between the sizes of the diploS/HIC-Rsb 284 regions in the eastern and western populations (Wilcoxon test, P-value=0.07; Figure $2853 \mathrm{~A})$.

286 We also tested whether distinct loci/genomic regions were affected by recent positive 287 selection in the two populations using linear models. While we found a significant 288 association between the density of genes under positive selection (S/HIC-Rsb and 289 S/HIC-Hscan outliers combined) in $100 \mathrm{~kb}$ windows in the eastern and western 290 populations on all chromosomes, all $\mathrm{R}^{2}$ were inferior to $1.307 \mathrm{e}-06$. This result 291 indicates that positive selection mostly affects distinct loci across the two populations 292 (as depicted on Figure 3B). Interestingly, we observed that some genomic regions 293 harbor higher density of genes under selection than the rest of the genome. Thus, 294 genes under positive selection appear to be non-randomly distributed along 295 chromosomes in the two populations (Figure 3B).

\section{Genes with extremely long coalescence time}

298 Balancing selection can be detected with coalescence approaches, as ancient alleles 299 are associated with older coalescence times (Charlesworth 2006). We used the 300 software ARGweaver to detect footprints of balancing selection, a method that has 301 robustly identified signatures of balancing selection in recently expanded human 302 populations (Rasmussen et al. 2014). Briefly, ARGweaver models the coalescent 303 process along chromosomes and across non-recombining haplotype blocks to infer 
304 their evolutionary history (see methods). It allows recovering several statistics such as 305 times to the most recent common ancestor (TMRCA, Figure 1C), which should be 306 increased near ancient alleles such as those under ancient balancing selection. By 307 doing so, we identified 72 regions harboring 115 genes under what will be referred to 308 as long-term balancing selection in the following (Table S3 for a list). This gene set 309 harbors a significant enrichment for genes involved in phosphorylation (Table 1).

Identification of candidate genes in known QTL regions

312 Combining association mapping and analyses of selection constitutes a powerful 313 approach to identify candidate genes and to address their selective regime. We 314 identified many candidate regions harboring resistance genes. As a proof of concept, 315 we therefore aimed at assessing whether regions identified as resistance loci against 316 known pathogens were also highlighted in our selection scans. In B. distachyon, the 317 genetic basis of resistance to the rust fungus Puccinia brachypodii has been 318 deciphered through a QTL mapping (Barbieri et al. 2012) which showed that leaf-rust 319 resistance is controlled by three main QTL located on chromosome 2 (from 320 nucleotide $37,949,269$ to $40,903,216$ ), 3 (from nucleotide 13,943,000 to $14,512,222$ ) 321 and 4 (from nucleotide 9,649,152 to 10,679,750). For the rest of the study, these 322 regions will be referred to as $\mathrm{QTL}_{\text {rust-2}}, \mathrm{QTL}_{\text {rust-3 }}$ and $\mathrm{QTL}_{\text {rust-4. }}$.

323 We screened these three regions for evidence of selection and found signals of 324 positive selection in $\mathrm{QTL}_{\text {rust-3 }}$ and $\mathrm{QTL}_{\text {rust-4. In }} \mathrm{QTL}_{\mathrm{rust}-3}$, we found that one gene 325 (Bradi3g16331: 14,502,928-14,503,290) belonged to the eastern high-confidence 326 gene set diploS/HIC-Rsb (Figure 4A, left panel). This gene is located $60 \mathrm{~kb}$ upstream 327 the QTL peak identified in this region (Barbieri et al. 2012) and codes for an 328 unknown protein. It is flanking a serine/threonine phosphatase (Bradi3g16320: 329 14,486,831- 14,488,838; Figure 4A, left panel) which was also identified as being 330 under selection in the eastern population with the Rsb test. The latter gene was 331 however not recovered by diploS/HIC but classified as a locus being linked to a 332 selected site (see methods). This $16 \mathrm{~kb}$ region spanned by Bradi3g16331 and 333 Bradi3g16320 may therefore be considered as a candidate for rust resistance.

334 The two adjacent Rsb signals in $\mathrm{QTL}_{\text {rust-4 }}$ reach their highest point into two R-genes 335 (Bradi4g10153: 9,807,879-9,812,927; and Bradi4g10171: 9,828,236-9,835,003; 336 Figure 4A, right panel). These two genes are located in the near vicinity of the QTL 
337 peak (estimated to be located at $9,806,000 \mathrm{~kb}$ ) and both belong to the eastern Rsb338 diploS/HIC high-confidence gene set (Figure 4A, right panel). Bradi4g10153 and

339 Bradi4g10171 constitute therefore further prime candidates for rust resistance.

340 Congruent with the signal detected by the Rsb test, we observed extended haplotypes

341 in the eastern population in both QTL $_{\text {rust-3 }}$ and QTL $_{\text {rust-4 }}$ (Figure 4B). The large

342 majority of the eastern accessions display the extended haplotype in these regions,

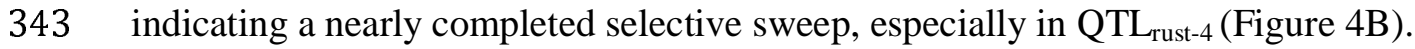

344 QTL $_{\text {rust-4 }}$ shows a striking enrichment for genes involved in defense response (P-value $345=8 \mathrm{E}-09)$ and in immune signaling processes such as phosphorylation $(\mathrm{P}$-value $=$ 346 5.6E-06). Among the 113 genes covered by QTL rust-4, 40 correspond either to a 347 gene with a NBS-LRR, a receptor-like protein kinase (RLK) or a F-box domains, 348 three types of genes that can confer resistance in plants. The presence of such a large gene clusters, as found in other regions of the genome (Table S2), further

350 demonstrates the importance of narrowing down candidate regions under selection to 351 top outlier genes for unbiased GO annotation.

352

\section{Discussion}

354 Assessing the time and spatial scales at which selection acts is a key to understand 355 how genetic diversity is maintained or lost through adaptation (Stinchcombe and 356 Hoekstra 2008; Fuller et al. 2015). In plants, and especially in grasses, this question 357 has been largely restricted to crops, which biases our understanding of evolutionary 358 processes that have shaped genomes in their natural ancestors and extant relatives. In 359 this study, we investigated the selective forces influencing adaptation in two 360 populations consisting of 44 natural accessions of the wild Mediterranean grass 361 B. distachyon. We found that ancient balancing and recent positive selection left 362 distinct signatures on specific gene categories, and that positive selection affects 363 distinct loci across the two populations. Importantly, our results support a role for 364 pathogens in driving population differentiation and confirm that GWSS constitute 365 effective approaches to pinpoint candidate genes as a complement to classical trait 366 mapping.

367 Time- and space-varying selection is shaping diversity in B. distachyon

368 The ending of the last glaciation period 10,000 years ago led to drastic and recent 369 changes of plant communities in Eurasia (Svenning et al. 2008; Binney et al. 2017). 
370 At that time, climate warmed and species expanded over Europe (Hewitt 1999).

371 Pollen-based studies show that vegetation expansion was fast, reaching up to $2 \mathrm{~km}$ per

372 year for some species (Hewitt 1999). To our knowledge, no fossil pollen records are

373 available for $B$. distachyon, which prevents reconstructing the geographical

374 distribution of this species before and during the last ice age. Yet a previous study

375 showed that the two populations analyzed here experienced population size reduction

376 during the last glaciation followed by a rapid expansion within the last 10,000 years

377 (Stritt et al., 2018). Even though unraveling the history of populations in southern

378 peninsulas is more complex than in northern regions (Hewitt 2000; Feliner 2011),

379 these results are congruent with the recent global postglacial recolonization of Europe

380 by plants (Hewitt 1999; Svenning et al. 2008; Binney et al. 2017) and imply that

381 B. distachyon populations had to adapt to newly colonized habitats in the recent past.

382 Balancing selection may have maintained ancestral polymorphisms over long periods

383 of time in natural populations of B. distachyon. As a support of balancing selection,

384 we identified an old set of shared polymorphisms with a coalescence approach.

385 B. distachyon populations encounter heterogeneous habitats (Lopez-Alvarez et al.,

386 2015) which could have provided selective pressure strong enough to maintain

387 polymorphisms over long periods of time within each population. Whether balancing

388 selection results from spatial heterogeneity or from negative frequency-dependent

389 selection remains yet to be investigated. Our results nevertheless suggest that

390 adaptation to climate after recolonization does not necessarily involve de novo

391 mutations, even after bottlenecks.

392 On the other hand, we also found evidence for positive selection acting on younger

393 polymorphisms. Our analysis also revealed that positive selection is targeting

394 different loci in the two populations and that these loci appear to be non-randomly

395 distributed along chromosomes. As recombination rate is relatively high in

396 B. distachyon (Huo et al. 2011) and since we observed a rather moderate linkage

397 disequilibrium in the two populations, we do not believe that this pattern is due to

398 extended linkage disequilibrium and the subsequent process of background selection

399 along large genomic regions (Cutter and Payseur 2013; Slotte 2014). Rather, the

400 peaks of selection we identified were narrow and allowed to pinpoint genes,

401 indicating that while $B$. distachyon is predominantly inbreeding, outcrossing events

402 must occur every now and then to limit extended linkage disequilibrium. This is also 
403 congruent with the slightly higher level of heterozygosity we observed here in

404 B. distachyon compared to other selfing plants such as A. thaliana (Platt et al. 2010).

405 Interestingly, the regions displaying signs of soft-sweep or ongoing selection tended

406 to be larger in the western population than in the one of the eastern cluster despite

407 slower LD decay in this latter group. This also indicates that selection may have acted

408 more recently in the western population, possibly due to the modern and rapid

409 expansion of these lineages in the Spanish peninsula (Stritt et al. 2018).

410 B. distachyon occurs exclusively around the Mediterranean rim which constitute a 411 mosaic of landscapes. Our results reveal that this variation in habitats lead to natural 412 selection which affected different genes and genomic regions across populations. As 413 we obtained enrichments for different GO terms in the eastern and western 414 populations, we also imply that contrasting abiotic and biotic factors are shaping 415 natural population diversity in different regions of the genome of B. distachyon 416 through positive selection. Even though we identified more genes under positive than 417 under balancing selection, it would be daring to conclude that the former selection 418 regime, less challenging to detect (Delph \& Kelly, 2014), is a predominant process 419 shaping diversity in this species. Rather, we believe that we provide here genomic 420 evidence that balancing selection also leads to the adaptation of $B$. distachyon 421 populations and that selection acted at different temporal scale.

\section{Pathogens as a potential driving force of population evolution}

424 Host-pathogen interactions lead to a strong coevolutionary dynamics and are 425 considered a major factor shaping diversity (Fumagalli et al. 2011; Karasov et al. 426 2014; Krattinger and Keller 2016a). Two main types of interaction have been 427 proposed. Under an arms race model, repeated innovation from both sides results in 428 repeated fixation of advantageous alleles (Brown and Tellier 2011). This interaction 429 can therefore lead to positive selection that can be detected by tests focusing on 430 extended haplotypes. The other type of interaction is often referred to as Red Queen 431 dynamics or trench warfare, where alleles involved in the interaction are recycled 432 through negative-frequency dependence and can therefore subsist for long periods of 433 time in populations through balancing selection (Brown and Tellier 2011). 
434 The plant immune system machinery is complex and is composed of two tiers of 435 extracellular and intracellular receptors (Krattinger and Keller 2016a; Krattinger and 436 Keller 2016b) that efficiently detect the presence of pathogens (for review Greeff et 437 al., 2012; Couto \& Zipfel, 2016; Eckardt, 2017). In this study, we found a significant 438 enrichment of signals of selection at genes involved in this first level of defense in the eastern population. More specifically, we found many of well-characterized R-genes, i.e. genes with NBS-LRR domains and involved in disease resistance (Mchale et al., 2006; Jacob et al., 2013; Liu et al., 2014; Couto \& Zipfel, 2016; Eckardt, 2017; 442 Ooijen et al., 2017), to be under ongoing or completed positive selection in the 443 eastern population. On the other hand, we also found additional R-genes and genes 444 involved in phosphorylation, a process especially important for immune signaling 445 response in plants (for review Park et al., 2012), to be under balancing selection. Our results are thus consistent with the two classical models of host-pathogens coevolution (Mondragón-palomino et al. 2002; Mchale et al. 2006; Gos et al. 2012; Mace et al. 2014; Zhong et al. 2015; Wu et al. 2017). Overall, and as shown in other organisms (Karasov et al. 2014; Fumagalli et al., 2011; Krattinger \& Keller, 2016a,b;

450 Bourgeois et al., 2017), our genome-wide approach suggests that pathogens may 451 constitute an important driving force of population and genome evolution in 452 B. distachyon.

453 The significant enrichments for R-genes and for genes associated to the process of 454 defense response in the gene sets under positive selection were only observed in the 455 eastern population. As we incorporated the demographic history to our analyses, we 456 conclude that the bottlenecks experienced by both populations did not extensively 457 bias our results and did not interfere with our conclusions on the importance of host458 pathogen interaction in the eastern population. The geographical origin of the 459 populations could, however, play a role in this observation. The Middle East is indeed 460 the center of origin of many grasses, including B. distachyon, and of their associated 461 pathogens (Wyand \& Brown, 2003; Stukenbrock et al., 2005; Opanowicz et al., 2008;

462 Hovmøller et al., 2011). Many studies found that both resistance genes in plants and 463 effector genes in pathogens can be organized in clusters evolving through an arm race 464 and resulting in a gene birth-and-death process (Michelmore and Meyers 1998; Dong 465 et al. 2015; Singh et al. 2015). Because centers of origin are usually associated to 466 higher diversity, it is therefore possible that a higher level of pathogen diversity drove 
467 selection at a larger number of resistance genes in the eastern population.

468

469

470

471

472

473

474

475

476

477

478

479

480

481

482

483

484

485

486

487

488

489

490

491

492

493

494

495

496

497

498

499

\section{GWSS as complementary approach to QTL and GWAS}

Disentangling the mechanisms that promote or prevent adaptation requires more integrated studies, using both experimentations in controlled conditions and methods to characterize genetic diversity in natural populations (Feder and Mitchell-olds 2003; Stinchcombe and Hoekstra 2008; Flood and Hancock 2017). Several studies used GWSS to validate genes functionally characterized or previously identified through GWAS and to propose stronger hypotheses on the mode of selection operating on traits relevant for adaptation (Roulin et al. 2016; Tang et al., 2007; Fumagalli et al., 2011; Bourgeois et al. 2017). Following this idea, we inspected three QTL regions responsible for the resistance of B. distachyon to the leaf-rust fungus Puccinia brachypodii, a natural pathogen of B. distachyon expected to exert selection on natural accessions.

For two of these QTL regions on chromosomes 3 and 4, we found signs of positive selection and reduced haplotype diversity in the eastern cluster in the vicinity of the QTL peaks, but no sign of balancing selection. These results strongly indicate that positive selection is shaping rust resistance in B. distachyon natural populations, as suggested in other species (Dodds \& Thrall, 2009; Chavan et al., 2015). On chromosome 3 , our analysis identified a $16 \mathrm{~kb}$ region spanned by an unknown protein and a serine/threonine phosphatase, a class of genes known for their role in defense response and stress signaling (País et al. 2009; Durian et al. 2016), to be putatively involved in resistance to rust. The region identified on chromosome 4 is more complex and consists of a cluster of resistance and stress signaling genes. Nevertheless, and while other genes display evidence of positive selection, two Rgenes that belong to the eastern high-confidence gene set co-localize with the peak of the QTL. Such genes have been shown to confer resistance to rust in other species (Bettgenhaeuser et al. 2014) and constitute further prime candidates for functional characterization.

$B$. distachyon is closely related to major crop cereals as well as to grass species used for biofuel production. Translating research from B. distachyon to plants of agronomical and economical interest will require a deeper understanding of the genetic architecture of traits involved in the response to environmental stresses. The 
500 molecular basis of tolerance to various abiotic stresses such as drought, salt and cold

501 has been investigated in this species (Luo et al. 2011; Manzaneda 2013; Carmo and

502 Charron 2014; Gordon et al. 2014; DesMarais and Juenger 2015; Sun et al. 2015; Mur

503 and Bosch 2016). Here, we also highlighted cadmium pollution as a potential factor of

504 positive selection in the eastern population. As pollution with heavy metals including

505 cadmium has been reported in Turkey in regions where accessions were collected for

506 this study (Bakirdere and Yaman 2008; Mor and Ceylan 2017), our results suggest

507 that $B$. distachyon could be used to investigate the tolerance to this stress. As genetic

508 transformation is highly efficient in this species relative to other grasses, we anticipate

509 that combining classical trait mapping analyses with GWSS will assist allele mining

510 for additional eco-responsive traits.

511

512

Conclusion

513 Our results revealed widespread signatures of natural selection at genes involved in

514 adaptation in $B$. distachyon and provide the community with a list of candidate genes

515 displaying strong signs of selection in natural populations. We also found that

516 pathogens may constitute an important driving force of genetic diversity and

517 evolution in this system. While we limited our analysis to classical point mutations,

518 recent studies showed that copy number variants (CNVs) and transposable element

519 polymorphisms are abundant across B. distachyon populations (Gordon et al., 2017;

520 Stritt et al., 2018). Hence, the important genomic resources currently developed in

521 this species open new avenues of research to further investigate the role of structural

522 variation in natural population evolution and adaptation. To date, B. distachyon

523 remains a classical model for research on grass genomics with a strong orientation

524 towards applied research. Thanks to the high quality of its reference genome and the

525 existence of large collections of freely available natural accessions collected from the

526 species native range, it also constitutes a prime system for studies in ecology,

527 population genomics and evolutionary biology.

528

529

530

531 


\section{Experimental procedures}

533 SNP calling, population structure and genetic diversity

534 We used paired-end Illumina sequencing data generated for 44 accessions of

535 B. distachyon (Gordon et al., 2017; Table S1 for information about the origin of the

536 accessions and sequencing effort) originating from Spain $(\mathrm{N}=16)$, France $(\mathrm{N}=1)$,

537 Turkey $(\mathrm{N}=23)$ and Iraq $(\mathrm{N}=4)$. Reads were aligned to the reference genome v2.0

538 with BWA-MEM (standard settings; Li, 2013). After removing duplicates with

539 Sambamba (Tarasov et al. 2017), single nucleotide polymorphisms (SNPs) were

540 called with Freebayes (Garrison and Marth 2016). The output was then filtered by

541 removing SNPs with more than 10 missing genotypes or more than 2 alleles, a quality

542 lower than 20 and a mean depth lower than 20 or higher than 200. Data were phased

543 using the software BEAGLE V4 (Browning and Browning 2007) using default

544 settings.

545 We then used the program Admixture (Alexander and Novembre 2009) to identify the

546 genetic structure of the two populations. The analysis was run for $\mathrm{K}$ values from 1 to

5475 , and the best model was determined as the model with the lowest cross-validation

548 error. Summary statistics such as within-population nucleotide diversity $(\pi)$ were

549 computed with the $\mathrm{R}$ package PopGenome (Pfeifer et al. 2014). Levels of

550 heterozygosity were calculated with VCFtools (Danecek et al. 2011).

551

552 Detecting positive selection associated to nearly completed sweeps

553 We used the Rsb test (Tang et al. 2007) to detect signatures of recent or almost

554 completed hard sweeps. This test detects haplotypes that are positively selected in one

555 population by estimating the length of haplotypes around each allele at a core SNP

556 and then comparing these lengths between populations. While the output of the test

557 provides $\mathrm{P}$-value of significance, it also indicates in which population a given allele is

558 under selection. Rsb statistics were computed for each SNP with the R package

559 rehh2.0 (Gautier et al. 2012) with default settings.

560

561 Detecting ongoing positive selection and soft-sweeps within populations

562 We used the software H-scan (Schlamp et al. 2016) to detect soft-sweep or loci under

563 incomplete ongoing positive selection. To do so, we calculated average pairwise

564 haplotypes lengths using the number of segregating sites spanned by each tract within 
565 each population. The H-scan statistics is expected to be larger as the number of 566 extended haplotypes increases in a population. We ran the method on eastern and 567 western accessions independently to detect soft- or incomplete selective sweeps 568 within each geographical group.

569

570

571

572

573

574

575

576

577

578

579

580

581

582

583

584

585

586

587

588

589

590

591

592

593

594

595

596

597

\section{Testing for functional clustering and subsequent filtering of GWWS results}

We first performed a GO annotation for the 32,712 genes annotated in the reference genome (version 2.1, https://phytozome.jgi.doe.gov) with Blast2GO (Conesa et al. 2005). We then controlled for potential gene clustering by following the procedure described in (Al-Shahrour et al., 2010). The entire gene set of the reference genome was split into windows of 50 consecutive genes. Windows were moved along chromosomes in steps of 25 genes to allow for half-window overlaps. Enrichment analyses of biological processes were then performed for all the generated windows with the R package GOstats (Falcon and Gentleman 2017) using Fisher's exact test. P-values were subsequently adjusted for multiple testing with a Benjamin-Hochberg correction. Regions were considered significantly enriched for a biological process when they displayed a corrected P-value $\leq 0.01$ and also harbored at least five genes associated to the given process.

Both the H-scan and the Rsb tests compute statistics at each SNP. To limit false positives, we first selected $10 \mathrm{~kb}$ windows displaying at least four significant SNPs within the top $1 \%$ outliers. Overlapping significant windows were merged. To limit the impact of gene clustering on the GO analysis, we then narrowed down the selected windows by keeping only the genes located at and around (-10\% of the peak value) each of the top $1 \%$ peaks of selection. These filtering criteria, however, were not applied to the outputs of ARGweaver and diploS/HIC, which are window-based approaches.

\section{Demographic history and filtering false positives}

We used the recently developed machine-learning algorithm diploS/HIC (Kern \& Schrider, 2018) to further control for the potentially confounding effects of demography on our outlier approach. To do so, genomic windows were simulated under neutrality, with a hard sweep or with a soft sweep. We trained the algorithm using a set of 3,000 coalescent simulations obtained with discoal (Kern and Schrider 
598 2016). We simulated 330kb windows each divided into 22 subwindows. Hard and soft 599 sweep examples consisted in windows with a sweep occurring in the two central $30 \mathrm{~kb}$ 600 subwindows. The adjacent subwindows are assigned as linked to selection.

601 We performed simulations for each of the two populations using demographic 602 trajectories inferred in a previous study (Stritt et al. 2018). Note that we rescaled these 603 estimates with a mutation rate of $1.4 \times 10-9 / \mathrm{bp} /$ generation instead of the previously 604 used $7 \times 10^{-9} / \mathrm{bp} /$ generation. We estimated this new mutation rate by aligning the 605 orthologs of 100 genes and using rice as an out-group (divergence estimated at 40My; 606 The International Brachypodium Consortium, 2010). We sampled selection 607 coefficients from an uniform prior $2 \mathrm{Ns} \sim(250,25000)$ for the eastern population and $6082 \mathrm{Ns} \sim(2000,200000)$ for the western population, to take into account the largest 609 estimate for present effective population size $(\mathrm{N})$ in the western cluster $(\mathrm{N} \approx 4,000,000)$ 610 compared to the eastern one $(\mathrm{N} \approx 500,000)$. We used a truncated exponential prior for 611 recombination rates encompassing the range $5.9 \times 10^{-9}-1.18 \times 10^{-7}$, values that are 612 between ten times lower and two times higher the rate of $5.9 \times 10^{-8}$ estimated from 613 controlled crosses (Huo et al. 2011).

614 To take into account the likely heterogeneity in effective population sizes and 615 mutation rates along the genome, we used priors for present effective population sizes 616 between twice lower and five times higher than the values estimated from the SMC++ 617 analysis for present times (Stritt et al. 2018). We conditioned on sweep completion 618 occurring between present time and shortly before populations expansion, around 10,000 generations ago. For soft sweeps, we used uniform priors on the initial 620 frequency of the adaptive variant of $(0,0.2)$. We took into account inbreeding by 621 assuming each individual contained two copies of the same haplotypes.

622 This set of simulated datasets was then used to train a supervised machine-learning 623 algorithm to differentiate between each category. Predictions on the actual genomic 624 dataset were then performed over subwindows. Since the statistics computed by 625 diploS/HIC are normalized across subwindows, they can remain flat if selection is 626 strong and erases diversity over the whole region to be classified. We therefore 627 classified genomic subwindows as selected, linked or neutral using two sizes (15 and $62850 \mathrm{~kb}$ ), in order to detect genomic regions displaying the strongest signals of selection.

629 The algorithm as well as a detailed tutorial are available at https://github.com/kern630 lab/diploSHIC. 
631 We finally used bedtools intersect (Quinlan and Hall 2010) to identify high-

632 confidence regions recovered by both the Rsb test and diploS/HIC or by both the $\mathrm{H}-$

633 scan test and diploS/HIC. Genes present in such high-confidence regions were

634 retrieved with bedtools intersect using the annotation of the genome of $B$. distachyon

635 v2.1 (https://phytozome.jgi.doe.gov).

636

637

Distribution of genes under positive selection along chromosomes

638 To compare the distribution along chromosomes of candidate genes for recent 639 positive selection (DiploS/HIC-Hscan and DiploS/HIC Rsb candidate genes 640 combined) in each population, we used linear models where the density of selected 641 genes along each chromosome identified in the eastern and western populations $642(100,000$ bins per chromosome $)$ were entered as variables. We eventually used circos 643 (Krzywinski et al. 2009) to visualize the density of genes under positive selection 644 along each chromosome.

645

646 Detecting ancient balancing selection with ancestral recombination graphs (ARG)

647 We used the software ARGWeaver to detect additional candidate regions for 648 balancing selection with a coalescence approach (Rasmussen et al. 2014). We 649 included in the analysis a subset of 12 accessions with high sequencing depth and 650 covering the largest geographical range (6 accessions from each population) to limit 651 computation time. We used a mutation rate of $1.4 \times 10-9 / \mathrm{bp} /$ generation and a 652 recombination rate of $5.9 \times 10-8 / \mathrm{bp} /$ generation. Note that we subsequently used an 653 outlier approach to identify the oldest polymorphisms present in the two populations 654 (top 1\% outliers). Therefore, potential biases inherent to the use of a molecular clock 655 do not affect our analysis. ARGWeaver is also flexible with regard to recombination 656 rate as it reconstructs ancestral recombination graphs and accommodates variable 657 recombination rates, effective population sizes and genealogies along the genome. 658 The algorithm was run for 1000 iterations, using 20 discretized time steps, a 659 maximum coalescence time of 3 million generations and a prior effective population 660 size of 100,000 individuals. This size was of the same order of magnitude as the 661 harmonic mean of effective population sizes over the last 500,000 generations 662 (spanning the recent population declines and expansions), as estimated in a previous 663 study (Stritt et al. 2018) using the algorithm SMC++ (Terhorst et al. 2017). 
665 GO annotation of genes under selection

666 For each high-confidence gene set and the gene set under balancing selection, we

667 extracted the genes located in the filtered regions with bedtools (Quinlan and Hall 668 2010). We then examined potential enrichment for biological processes for each of 669 the selected gene sets with the R package GOstats (Falcon and Gentleman 2017) 670 using the Fisher's exact test. Gene sets were considered significantly enriched for a 671 biological process when they displayed a P-value $\leq 0.01$ and harbored at least five 672 genes associated to the given process. The ancestor and child terms of each significant 673 process were determined using QuickGO (http://www.ebi.ac.uk/QuickGO) and used 674 to simplify Fisher test outputs and keep non-redundant terms.

675 We also retrieved from each gene set under selection the number of genes containing 676 a NBS-LRR domain, and annotated as R-genes in version 2.1 of the genome. We then 677 estimated whether these gene sets were enriched for R-genes compared to the rest of 678 the genome with Fisher's exact test in R (R development core team, 2017).

679

680 QTL for leaf-rust resistance validation

681 A QTL analysis performed in B. distachyon revealed three genomic regions involved 682 in the resistance to P. brachypodii (Barbieri et al. 2012). The coordinates of these 683 three QTL were extracted from (Barbieri et al. 2012) from v.2.1 of the B. distachyon 684 reference genome. We then assessed weather those three regions were identified as 685 outliers in at least one of the tests of selection or in the high-confidence gene set. We 686 further visualized the extension of haplotypes at candidate regions using the 687 bifurcation.diagram() function of the rehh2.0 R package.

688

689 Acknowledgements

690 We thank the Genetic Diversity Center-ETH Zurich for providing access the Euler 691 high-performance cluster. This research was carried out on the High Performance 692 Computing resources at New York University Abu Dhabi and ETH-Zurich. We also 693 would like to thank Beat Keller's group as well as Mahendra Mariadassou for their 694 advices during the elaboration of the study. This work is supported by the Ambizione 695 program of the Swiss National Science Foundation (PZ00P3_154724). The work 696 conducted by the U.S. Department of Energy Joint Genome Institute, a DOE Office of 697 Science User Facility, is supported under Contract No. DE-AC02-05CH11231. 


\section{Availability of data and materials}

699 The raw vcf file and the raw outputs of each GWWS will be archive upon acceptance

700 of the manuscript. All whole-genome sequences data are available at the NCBI

701 Sequence Read archive (SRA available in Gordon et al., 2017).

702

\section{Conflict of interest}

704 There is no conflict of interest issue related to this work.

705

706 Supporting Information

707 Figure S1: Admixture analysis

708 Cross-validation plot.

709 Figure S2: Confusion matrices for diploS/HIC.

710 The trained algorithm was applied on datasets simulated under a coalescent

711 framework, and the proportion of simulations correctly assigned to their category was

712 estimated. The location of the classified subwindows relative to the sweep is shown

713 vertically and the predicted class for each subwindow is shown horizontally. Each

714 row corresponds to 3,000 simulations.

715 Table S1: Geographical coordinates of the 44 accessions and sequencing effort.

716 Table S2: Significant GO terms in each 50 gene-window of the reference genome.

717 Table S3: List of genes under selection. 
719

720

721

722

723

724

725

726

727

728

729

730

731

732

733

734

735

736

737

738

739

740

741

742

743

744

745

746

747

748

749

750

751

752

753

754

755

756

757

758

759

760

761

\section{References}

Al-Shahrour, Minguez P, Marques-Bonet T, Gazave E, Navarro A, Dopazo J. 2010. Selection upon Genome Architecture $\square$ : Conservation of Functional Neighborhoods with Changing Genes. PLoS computational biology 6: e1000953.

Alexander DH, Novembre J. 2009. Fast Model-Based Estimation of Ancestry in Unrelated Individuals. Genome research 19: 1655-1664.

Bakirdere S, Yaman M. 2008. Determination of lead, cadmium and copper in roadside soil and plants in Elazig, Turkey. environmental monitoring Assessment 136: 401-410.

Barbieri M, Marcel TC, Niks RE, Francia E, Pasquariello M, Mazzamurro V, Garvin DF. 2012. QTLs for resistance to the false brome rust Puccinia brachypodii in the model grass. Genome 163: 152-163.

Bettgenhaeuser J, Gilbert B, Ayliffe M, Moscou MJ. 2014. Nonhost resistance to rust pathogens - a continuation of continua. frontiers in plant science 5: 1-15.

Binney H, Edwards M, Macias-fauria M, Lozhkin A, Anderson P, Kaplan JO, Andreev A, Bezrukova E, Blyakharchuk T, Jankovska V, et al. 2017. Vegetation of Eurasia from the last glacial maximum to present $\square$ : Key biogeographic patterns. Quaternary Science Reviews 157: 80-97.

Bischoff A, Crémieux L, Smilauerova M, Lawson CS, Mortimer SR, Dolezal J, Edwards AR, Brook AJ, Macel M, Leps JAN, et al. 2006. Detecting local adaptation in widespread grassland species - the importance of scale and local plant community. journal of ecology 94: 1130-1142.

Bourgeois Y, Roulin AC, Müller K, Ebert D. 2017. Parasitism drives host genome evolution: Insights from the Pasteuria ramosa - Daphnia magna system. Evolution 71:1106-13.

Brown JKM, Tellier A. 2011. Plant-parasite coevolution: bridging the gap between genetics and ecology. Annual Review of Phytopathology 49: 345-67.

Browning SR, Browning BL. 2007. Rapid and Accurate Haplotype Phasing and Missing-Data Inference for Whole-Genome Association Studies By Use of Localized Haplotype Clustering. the american journal of human genetics 81: 1084-1097.

Carmo S Do, Charron J. 2014. Comparative analysis of the cold acclimation and freezing tolerance capacities of seven diploid Brachypodium distachyon accessions. Annals of botany 113: 681-693.

Ceballos, G., Davidson, A., List, R., Pacheco, J., Manzano-Fisher, P., SantosBarrera, G., Cruzado J. 2010. Rapid Decline of a Grassland System and Its Ecological and Conservation Implications. PloS one 5: e8562.

Charlesworth D. 2006. Balancing selection and its effects on sequences in nearby genome regions. PLoS Genetics 2: e64.

Chavan S, Gray J, Smith SM. 2015. Diversity and evolution of Rp1 rust resistance genes in four maize lines. Theoretical and Applied Genetics 128: 985-998.

Clayton WD. 1981. Evolution and Distribution of Grasses. Annals of the Missouri Botanical Garden 68: 5-14. 
Conesa A, Götz S, García-Gómez JM, Terol J, Talón M, Robles M. 2005. Blast2GO: a universal tool for annotation, visualization and analysis in functional genomics research. Bioinformatics 21: 3674-6.

Couto D, Zipfel C. 2016. Regulation of pattern recognition receptor signalling in plants. nature reviews immunology 16: 537-552.

Cutter AD, Payseur BA. 2013. Genomic signatures of selection at linked sites $\square$ : unifying the disparity among species. Nature reviews Genetics 14: 262-274.

Danecek P, Auton A, Abecasis G, Albers CA, Banks E, Depristo MA, Handsaker RE, Lunter G, Marth GT, Sherry ST, et al. 2011. The variant call format and VCFtools. Bioinformatics 27: 2156-2158.

Dell'Acqua MD, Zuccolo A, Tuna M, Gianfranceschi L, Pè ME. 2014. Targeting environmental adaptation in the monocot model Brachypodium distachyon $\square$ : a multi-faceted approach. BMC Genomics 15: 801.

Delph LF, Kelly JK. 2014. On the importance of balancing selection in plants Lynda. New phytologist 201: 1-22.

Des Marais DL, Juenger TE. 2015. Brachypodium and the Abiotic Environment. In: Vogel J, editor. Genetics and Genomics of Brachypodium, 291-311.

Dodds P, Thrall P. 2009. Recognition events and host-pathogen co-evolution in gene-for- gene resistance to flax rust. Functional plant biology 36: 395-408.

Dong S, Raffaele S, Kamoun S. 2015. ScienceDirect The two-speed genomes of filamentous pathogens $\square$ : waltz with plants. Current Opinion in Genetics \& Development 35: 57-65.

Durian G, Rahikainen M, Alegre S, Brosché M. 2016. Protein Phosphatase 2A in the Regulatory Network Underlying Biotic Stress Resistance in Plants. frontiers in immunology 7: 1-17.

Eckardt NA. 2017. The Plant Cell Reviews Plant Immunity $\square$ : Receptor-Like Kinases, ROS-RLK Crosstalk, Quantitative Resistance, and the Growth / Defense Trade-Off. Plant cell 29: 601-602.

Falcon SÃ, Gentleman R. 2017. Using GOstats to test gene lists for GO term association. Bioinformatics 23: 257-258.

Feder ME, Mitchell-olds T. 2003. Evolutionary and ecological functional genomics. Nature reviews Genetics 4: 649-655.

Feliner GN. 2011. Southern European glacial refugia $\square$ : A tale of tales. taxon 60: 365-372.

Fijarczyk A, Babik A. 2015. Detecting balancing selection in genomes $\square$ : limits and prospects. Molecular ecology 24: 3529-3545.

Flood J, Hancock AM. 2017. ScienceDirect The genomic basis of adaptation in plants. Current opinion in plant biology 36: 88-94.

Fuller ZL, Niño EL, Patch HM, Bedoya-Reina OC, Baumgarten T, Muli E, Mumoki F, Ratan A, McGraw J, Frazier M, et al. 2015. Genome-wide analysis of signatures of selection in populations of African honey bees (Apis mellifera) using new web-based tools. BMC genomics 16: 518.

Fumagalli M, Sironi M, Pozzoli U, Ferrer-admettla A, Pattini L. 2011. Signatures 
805

806

807

808

809

810

811

812

813

814

815

816

817

818

819

820

821

822

823

824

825

826

827

828

829

830

831

832

833

834

835

836

837

838

839

840

841

842

843

844

845

846

847 of Environmental Genetic Adaptation Pinpoint Pathogens as the Main Selective Pressure through Human Evolution. PLoS genetics 7: e1002355.

Garrison E, Marth G. 2016. Haplotype-based variant detection from short-read sequencing. Available at:https://arxiv.org/abs/1207.3907

Gautier M, Vitalis R, Baillarguet D, Cedex F-M. 2012. rehh $\square$ : an R package to detect footprints of selection in genome-wide SNP data from haplotype structure. Bioinformatics 28: 1176-1177.

Gibson DJ. 2009. Grasses and grassland ecology (Oxford university press, Ed.).

Gordon SP, Contreras-Moreira B, Woods DP, Des Marais DL, Burgess D, Shu S, Stritt C, Roulin AC, Schackwitz W, Tyler et al. 2018. Extensive gene content hetlvariation in the Brachypodium distachyon pan-genome correlates with phenotypic variation. Nature Communications 8: 2184

Gordon SP, Priest H, Marais DL Des, Schackwitz W, Figueroa M, Martin J, Jennifer N, Tyler L, Lee C, Bryant D, et al. 2014. Genome diversity in Brachypodium distachyon $\square$ : deep sequencing of highly diverse inbred lines. Plant Journal 79: 361-374.

Gos G, Slotte T, Wright SI. 2012. Signatures of balancing selection are maintained at disease resistance loci following mating system evolution and a population bottleneck in the genus Capsella. BMC Evolutionary Biology 12: 152.

Greeff C, Roux M, Mundy J, Petersen M. 2012. Receptor-like kinase complexes in plant innate immunity. frontiers in plant scienceontiers in plant science 3: 1-7.

Groves RH. 2000. Temperate grasslands of the southern hemisphere. In: Jacobs SWL, Everett J, editors. Grasses Systematics and Evolution, 3781-3791.

Hassl RJ, Payseur BA. 2016. Detecting selection in natural populations: Making sense of genome scans and towards alternative solutions. Molecular Ecology 25: $5-23$.

Helm A, Oja T, Saar L, Takkis K, Talve T, Pa M. 2009. Human influence lowers plant genetic diversity in communities with extinction debt. journal of ecology $\mathbf{9 7}$ 1329-1336.

Hermisson J. 2009. Who believes in whole-genome scans for selection $\square$ ? Heredity 103: $283-284$.

Hewitt GM. 1999. Post-glacial re-colonization of European biota. Biological journal of the Linnean society 68: 87-112.

Hewitt G. 2000. The genetic legacy of the Quaternary ice ages. Nature 405: 907-913.

Hovmøller MS, Sørensen CK, Walter S, Justesen AF. 2011. Diversity of Puccinia striiformis on Cereals and Grasses. Annual Review of Phytopathology 49: 197-220.

Huang J, Pray C, Rozelle S. 2002. Enhancing the crops to feed the poor. Nature 418: $678-684$.

Huo N, Garvin DF, You FM, Luo SMM, Gu YQ, Lazo GR, Philip J. 2011. Comparison of a high-density genetic linkage map to genome features in the model grass Brachypodium distachyon. Theoretical and Applied Genetics 123: 455-464.

Jacob F, Vernaldi S, Maekawa T. 2013. Evolution and conservation of plant NLR functions. frontiers in immunology 4: 1-16. 
848

849

850

851

852

853

854

855

856

857

858

859

860

861

862

863

864

865

866

867

868

869

870

871

872

873

874

875

876

877

878

879

880

881

882

883

884

885

886

887

888

889

890
Karasov TL, Horton MW, Bergelson J. ScienceDirect Genomic variability as a driver of plant - pathogen coevolution $\square$ ? Current Opinion in Plant Biology 18: 24-30.

Kern AD, Schrider DR. 2016. Discoal: flexible coalescent simulations with selection. Bioinformatics 32:3839-3841.

Kern AD, Schrider DR. 2018. diploS/HIC: An Updated Approach to Classifying

Selective Sweeps. G3; Genes|Genomes|Genetics 8: 1959-1970.

Kiviniemi K, Eriksson O. 2002. Size-related deterioration of semi-natural grassland fragments in Sweden. diversity and distribution 8: 21-29.

Krattinger SG, Keller B. 2016a. Tansley review Molecular genetics and evolution of disease resistance in cereals. New phytologist 212: 320-332.

Krattinger SG, Keller B. 2016b. Trapping the intruder — immune receptor domain fusions provide new molecular leads for improving disease resistance in plants. Genome Biology 17:23.

Krzywinski M, Schein J, Birol I, Connors J, Gascoyne R, Horsman D, Jones SJ, Marra M a. 2009. Circos: an information aesthetic for comparative genomics. Genome research 19: 1639-45.

Latta RG. 2009. Testing for local adaptation in Avena barbata $\square$ : a classic example of ecotypic divergence. Molecular Ecology 18: 3781-3791.

Li H. 2013. Aligning sequence reads, clone sequences and assembly contigs with BWA-MEM. preprint arXiv:1303.3997.

Liu W, Frick M, Huel R, Nykiforuk CL, Wang X, Gaudet DA, Eudes F, Conner RL, Kuzyk A, Chen Q, et al. 2014. The Stripe Rust Resistance Gene Yr10 Encodes an Evolutionary-Conserved and Unique CC - NBS - LRR Sequence in Wheat. Molecular Plant 7: 1740-1755.

Lopez-Alvarez D, Manzaneda AJ, Rey P, Giraldo P, Benavente E, Allainguillaume $\mathbf{J}$ et al. 2015. Environmental niche variation and evolutionary diversifi cation of the Brachypodium distachyon grass complex species in their native. American journal of botany 102: 1073-1088.

Luo N, Liu J, Yu X, Jiang Y. 2011. Natural variation of drought response in Brachypodium distachyon. physiologia plantarum 141: 19-29.

Mace E, Tai S, Innes D, Godwin I, Hu W, Campbell B, Gilding E, Cruickshank A, Prentis P, Wang J, et al. 2014. The plasticity of NBS resistance genes in sorghum is driven by multiple evolutionary processes. BMC plant biology 14: 253.

Manzaneda AJ, Rey PJ, Bastida JM, Weiss-Lehman C, Raskin E, Mitchell-Olds T. 2013. Environmental aridity is associated with cytotype segregation and polyploidy occurrence in Brachypodium distachyon (Poaceae) Antonio. New phytologist 193: 797-805.

Mchale L, Tan X, Koehl P, Michelmore RW. 2006. Plant NBS-LRR proteins $\square$ : adaptable guards. Genome Biology 7: 212.

Messer PW. 2015. H-scan $\square$ : Detecting hard and soft sweeps in population genomic data. 3: 1-4.

Michelmore RW, Meyers BC. 1998. Clusters of Resistance Genes in Plants Evolve 
891

892

893

894

895

896

897

898

899

900

901

902

903

904

905

906

907

908

909

910

911

912

913

914

915

916

917

918

919

920

921

922

923

924

925

926

927

928

929

930

931

932

933 by Divergent Selection and a Birth-and-Death Process. Genome research 530: 1113-1130.

Mitchell-Olds T, Willis JH, Goldstein DB. 2007. Which evolutionary processes influence natural genetic variation for phenotypic traits? Nature reviews Genetics 8: $845-56$.

Mondragón-palomino M, Meyers BC, Michelmore RW, Gaut BS. 2002. Patterns of Positive Selection in the Complete NBS-LRR Gene Family of Arabidopsis thaliana. Genome research 12: 1305-1315.

Mor F, Ceylan S. 2017. Cadmium and lead contamination in vegetables collected from industrial, traffic and rural areas in Bursa Province, Turkey. Food Additives \& Contaminants $\square$ 25: 611-615.

Morris GP, Ramu P, Deshpande SP, Hash CT, Shah T, Upadhyaya HD. 2013. Population genomic and genome-wide association studies of agroclimatic traits in sorghum. Proceedings of the National Academy of Sciences 110: 453-458.

Mur LAJ, Bosch M. 2016. Linking Dynamic Phenotyping with Metabolite Analysis to Study Natural Variation in Drought Responses of Brachypodium distachyon. frontiers in plant science 7: 1-15.

Nielsen R. 2005. Molecular Signatures of Natural Selection. Annual Review of Genetics. 39: 197-218.

Ooijen G Van, Mayr G, Kasiem MMA, Albrecht M, Cornelissen BJC, Takken FLW. 2017. Structure - function analysis of the NB-ARC domain of plant disease resistance proteins. jouranl of experimental botany 59: 1383-1397.

Opanowicz M, Vain P, Draper J, Parker D, Doonan JH. 2008. Brachypodium distachyon $\square$ : making hay with a wild grass. Trends in Plant Science 13: 172-177.

País SM, Téllez-iñón MT, Capiati DA. 2009. Serine / threonine protein phosphatases type $2 \mathrm{~A}$ and their roles in stress signaling. Plant signaling and behaviour 4: 1013-1015.

Park C, Caddell DF, Ronald PC. 2012. Protein phosphorylation in plant immunity $\square$ : insights into the regulation of pattern recognition receptor-mediated signaling. frontiers in plant science 3: 1-9.

Pavlidis P, Jensen JD, Stephan W, Stamatakis A. 2012. A Critical Assessment of Storytelling $\square$ : Gene Ontology Categories and the Importance of Validating Genomic Scans Research article. Molecular biology and evolution 29: 3237-3248.

Pfeifer B, Wittelsbürger U, Ramos-Onsins SE, Lercher MJ. 2014. PopGenome: An efficient swiss army knife for population genomic analyses in R. Molecular Biology and Evolution 31: 1929-1936.

Platt A, Horton M, Huang YS, Li Y, Anastasio AE, Mulyati NW, Bossdorf O, Byers D, Donohue K, Dunning M, et al. 2010. The Scale of Population Structure in Arabidopsis thaliana. plos genetics 6: e1000843.

Quinlan AR, Hall IM. 2010. BEDTools $\square$ : a flexible suite of utilities for comparing genomic features. Bioinformatics 26: 841-842.

R development core team. 2017. R: A language and environment for Statistical Computing. 
934 Rasmussen MD, Hubisz MJ, Gronau I, Siepel A. 2014. Genome-Wide Inference of

935

936

937

938

939

940

941

942

943

944

945

946

947

948

949

950

951

952

953

954

955

956

957

958

959

960

961

962

963

964

965

966

967

968

969

970

971

972

973

974

975

976

977

Ancestral Recombination Graphs. PLoS genetics 10: e1004342.

Roulin A, Bourgeois Y, Stiefel U, Walser J-C, Ebert D. A photoreceptor underlies natural variation in Daphnia magna diapause induction. Molecular Biology and Evolution. 12:3194-3204.

Savolainen O, Lascoux M, Merilä J. 2013. Ecological genomics of local adaptation. Nature reviews Genetics 14: 807-820.

Schlamp F, Van Der Made J, Stambler R. 2016. Evaluating the performance of selection scans to detect selective sweeps in domestic dogs. Molecular Ecology 25: 342-356.

Schrider DR, Kern AD. 2016. S/HIC $\square$ : Robust Identification of Soft and Hard Sweeps Using Machine Learning. PLoS genetics 12(3): 1-31.

Singh S, Chand S, Singh NK, Sharma TR. 2015. Genome-Wide Distribution, Organisation and Functional Characterization of Disease Resistance and Defence Response Genes across Rice Species. Plos one 10:e125964

Slavov GT, Nipper R, Robson P, Farrar K, Allison GG, Bosch M, Clifton-brown JC, Donnison IS, Jensen E. 2014. Genome-wide association studies and prediction of 17 traits related to phenology, biomass and cell wall composition in the energy grass Miscanthus sinensis. New phytologist 201: 1227-1239.

Slotte T. 2014. The impact of linked selection on plant genomic variation. briefings in functional genomics 13: 268-275.

Stamatakis A, Alachiotis N, Pavlidis P, Daniel Z. 2013. SweeD $\square$ : LikelihoodBased Detection of Selective Sweeps in Thousands of Genomes. Molecular biology and evolution 30: 2224-2234.

Stinchcombe JR, Hoekstra HE. 2008. Combining population genomics and quantitative genetics: finding the genes underlying ecologically important traits. Heredity 100: 158-170.

Stritt C, Gordon SP, Wicker T, Vogel JP, Roulin AC. 2018. Recent activity in expanding populations and purifying selection have shaped transposable element landscapes across natural accessions of the Mediterranean grass Brachypodium distachyon. Genome Biology and Evolution 10:304-318.

Stromberg C. 2011. Evolution of Grasses and Grassland Ecosystems. Annual review of earth and planetary science 39: 517-544.

Stukenbrock EH, Banke S, Javan-nikkhah M, Mcdonald BA. 2005. Origin and Domestication of the Fungal Wheat Pathogen Mycosphaerella graminicola via Sympatric Speciation. Molecular biology and evolution 24: 398-411.

Sun J, Hu W, Zhou R, Wang L. 2015. The Brachypodium distachyon BdWRKY36 gene confers tolerance to drought stress in transgenic tobacco plants. Plant cell reports 34: 23-35.

Svenning J, Normand S, Kageyama M. 2008. Glacial refugia of temperate trees in Europe $\square$ : insights from species distribution modelling. journal of ecology 96: 1117-1127.

Tang K, Thornton KR, Stoneking M. 2007. A New Approach for Using Genome Scans to Detect Recent Positive Selection in the Human Genome. PLoS biology 
978

979

980

981

982

983

984

985

986

987

988

989

990

991

992

993

994

995

996

997

998

999

1000

1001

1002

1003

1004

1005

1006

1007

1008

1009

1010 5:e171

Tarasov A, Vilella AJ, Cuppen E, Nijman IJ, Prins P. 2017. Genome analysis Sambamba $\square$ : fast processing of NGS alignment formats. Bioinformatics 31: 20322034.

Teixeira C, Filippo C De, Key FM, Andre AM. 2014. ScienceDirect Advantageous diversity maintained by balancing selection in humans. Current opinion in genetics \& development 29: 45-51.

The international brachypodium Consortium. 2010. Genome sequencing and analysis of the model grass Brachypodium distachyon. Nature 463: 763-8.

Tyler AL, Lee SJ, Young ND, Deiulio GA, Benavente E, Reagon M, Sysopha J, Baldini RM, Troì A, Hazen SP, et al. 2016. Population structure in the model grass Brachypodium distachyon is highly correlated with flowering differences across broad geographic areas. The Plant Genome 9:1-55.

Wadgymar SM, Lowry DB, Gould BA, Byron CN, Mactavish RM, Anderson JT. 2017. Identifying targets and agents of selection: innovative methods to evaluate the processes that contribute to local adaptation. Methods in Ecology and Evolution 8: $738-749$.

Wallace JS, Batchelor CH. 1997. Managing water resources for crop production. Philosophical transactions of the Royal Society of London. Series B, Biological sciences 352: 937-947.

Wu Q, Han T, Chen X, Chen J, Zou Y, Li Z, Xu Y. 2017. Long-term balancing selection contributes to adaptation in Arabidopsis and its relatives. Genome biology 18: 217.

Wyand RA, Brown K. 2003. Genetic and forma specialis diversity in Blumeria graminis of cereals and its implications for host-pathogen co-evolution. Molecular plant pathology 4: 187-198.

Zhong Y, Li Y, Huang K, Cheng Z. 2015. Species-specific duplications of NBSencoding genes in Chinese chestnut (Castanea mollissima). Scientific reports 5: 16638. 


\section{Figures}

1012

1013

1014

1015

1016

1017

1018

1019

1020

1021

1022

1023

1024

1025

1026

1027

1028

1029

1030

1031

1032

1033

1034

1035

1036

1037

1038

1039

1040

1041

1042

1043

1044

1045

Figure 1: Schematic representation of the regimes of selection analyzed in this study

A) Cross-population tests: in case of positive selection on a new mutation, SNPs linked to the advantageous mutation will also rise in frequency, leading to extended haplotypes in the vicinity of the selected mutation. Cross-population tests detect haplotypes that are positively selected in one population by estimating the length of haplotypes around each allele at a core SNP, then comparing these lengths between populations. Such tests are designed to identify hard-sweep, i.e. region where one haplotype reached near fixation B) Within-population tests: these scans measure haplotype length at a focal locus and compare it to the average length of haplotypes along the genome in a given population. They allow recovering regions with haplotypes longer than average (top 1\% outliers) and can identify loci under selection independently of the number of haplotypes or of the completeness of the sweep C) Coalescence approach: balancing selection can maintain shared polymorphisms across populations due the habitat heterogeneity or negative frequency-dependent selection. Associated to old alleles, long-term balancing selection can be detected by calculating time to most recent common ancestor (TMRCA), which should increase at loci maintained by selection over long period of time.

\section{Figure 2: Geographical origin and genetic diversity of the 44 accessions}

A) Geographical origin of the 44 accessions used in the study. The asterisk indicates the origin of the reference accession $\mathrm{Bd} 21$ B) Structure plot $(\mathrm{K}=2)$ displaying the limited level of admixture between the eastern and western population. Each bar represents the genetic data of one accession. An accession presents some admixture when the bar displays different colors. The height of the bar is proportional to the admixture level C) Nucleotide diversity ( $\pi$ in $5 \mathrm{~kb}$ windows) within the eastern and western populations $\mathrm{D}) \mathrm{LD}$ decay in the eastern and western populations.

\section{Figure 3: Density of genes under selection}

A) Number of genes per high-confidence region for the high-confidence gene sets B) Circular representation of gene density along chromosomes (i) density of genes under positive selection in the eastern population (DiploS/HIC-Rsb and DiploS/HIC-Hscan combined), scaled from 0 to 20 genes per $100 \mathrm{~kb}$ (ii) density of genes under positive 
1046 selection in the western population (DiploS/HIC-Rsb and DiploS/HIC-Hscan

1047 combined), scaled from 0 to 20 genes per $100 \mathrm{~kb}$ (iii) overall gene density, scaled

1048 from 0 to 40 genes per $100 \mathrm{~kb}$.

1049

1050

Figure 4: QTL involved in resistance to rust

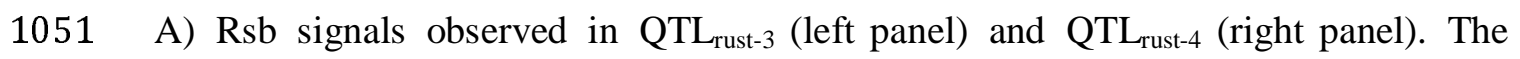
1052 dashed line indicates the $1 \%-\log _{10}$ (Rsb P-values) outlier threshold. Note that in order 1053 to reduce the size of the figure, points with $-\log _{10}$ (Rsb P-values) values $<1$ are not 1054 displayed. Grey boxes display the position of regions detected by diploS/HIC. Red 1055 bars display the position of genes detected by the Rsb test B) Haplotype bifurcation 1056 diagram in QTL sub-regions displaying strong of selection. Left panel: zoom in on 1057 chromosome 3; right panel: zoom in on chromosome 4. The diagrams visualize the 1058 breakdown of LD at increasing distances from the selected focal SNP, displayed by a 1059 vertical black line. Each horizontal line represents a haplotype. Each accession is 1060 represented by two lines, one for each haplotype. Horizontal blue lines are merged 1061 when two accessions share the same haplotype. The thickness of the line is therefore 1062 correlated to the number of accessions sharing the same haplotype. 
bioRxiv preprint doi: https://doi.org/10.1101/246090; this version posted June 17, 2018. The copyright holder for this preprint (which was not certified by peer review) is the author/funder, who has granted bioRxiv a license to display the preprint in perpetuity. It is made available under

Table 1: significant GO term associated to each teest of selection

\begin{tabular}{llc}
\hline \hline Test & Significant GO process & Pvalue \\
\hline TMRCA & Phosphorylation & $3.13 \mathrm{E}-08$ \\
\hdashline DiploS/HIC-Rsb East & $\begin{array}{c}\text { Response to stress including: } \\
\text { - defense response }\end{array}$ & $\begin{array}{l}5.00 \mathrm{E}-04 \\
9.4 \mathrm{E}-04\end{array}$ \\
\hline \multirow{2}{*}{ DiploS/HIC-Rsb West } & Amide transport & $5.00 \mathrm{E}-03$ \\
\hline \multirow{2}{*}{ DiploS/HIC-Hscan East } & Response to cadmium ion & $6.6 \mathrm{E}-04$ \\
& Small molecule metabolic process & $3.8 \mathrm{E}-03$ \\
\hline & Pyruvate metabolic process & $3.80 \mathrm{E}-03$ \\
DiploS/HIC-Hscan West & Carbohydrate derivative metabolic process & $3.80 \mathrm{E}-03$ \\
& Phospholipid metabolic process & $3.40 \mathrm{E}-03$ \\
\hline \hline
\end{tabular}




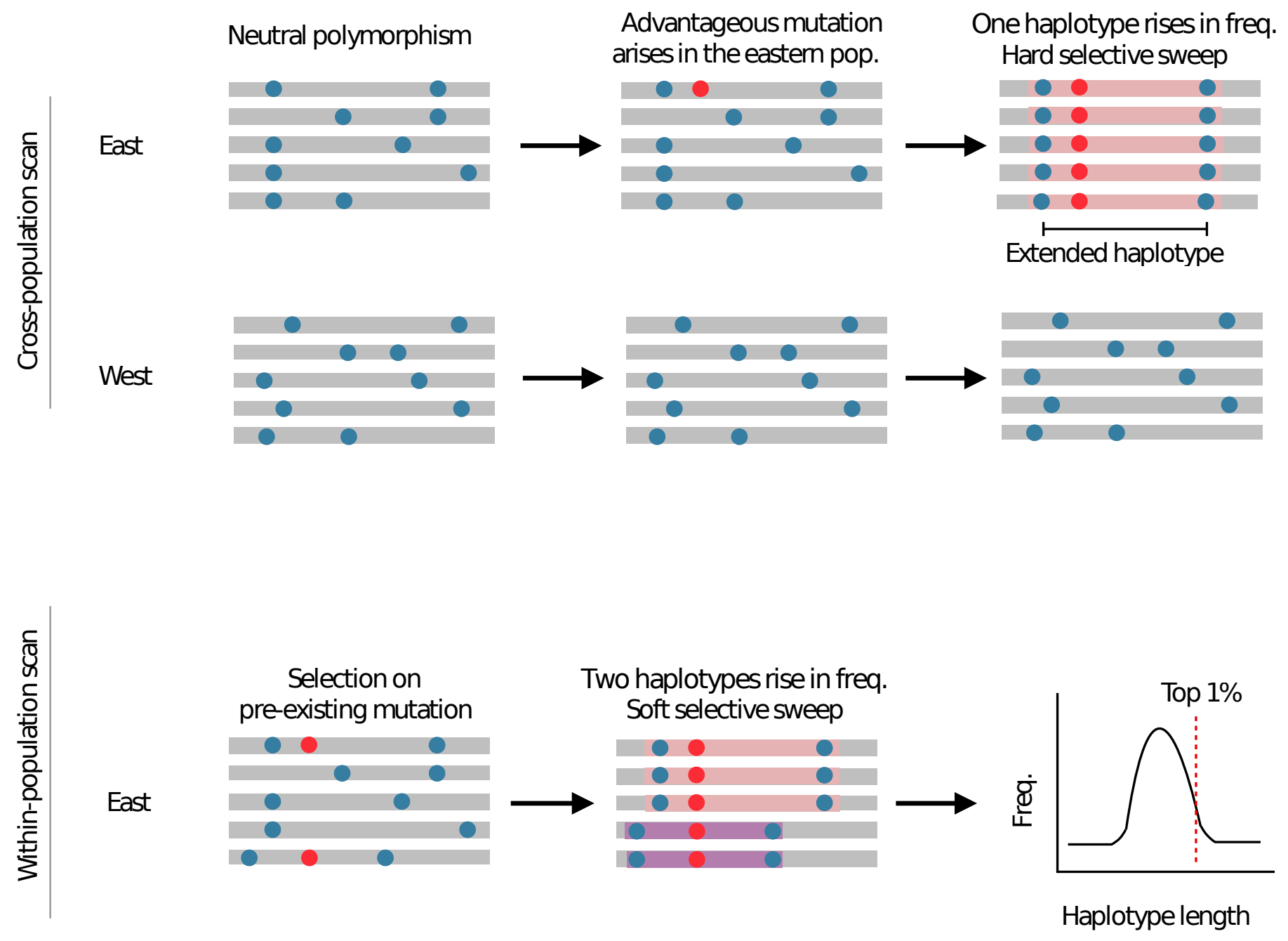

C
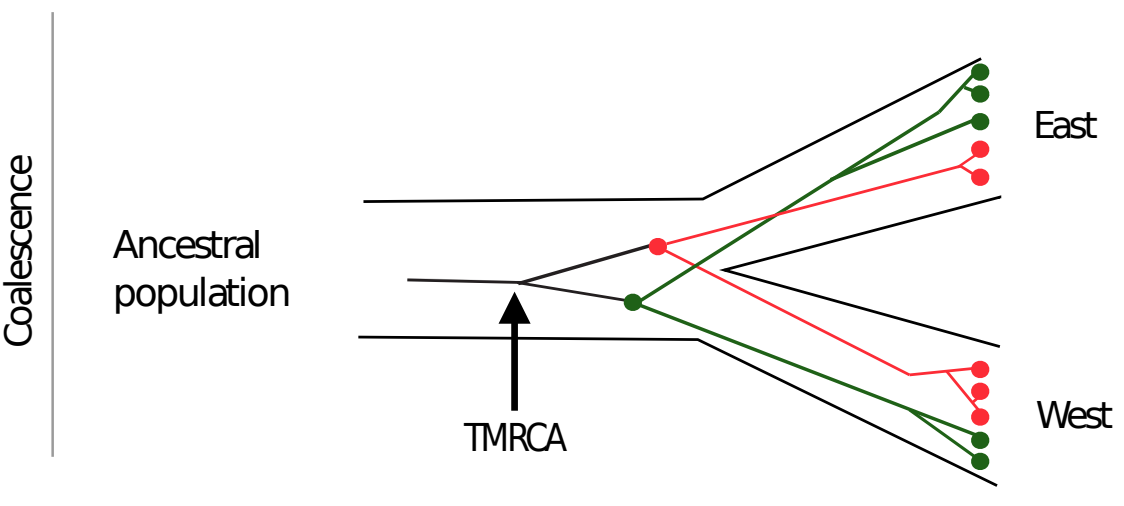
C
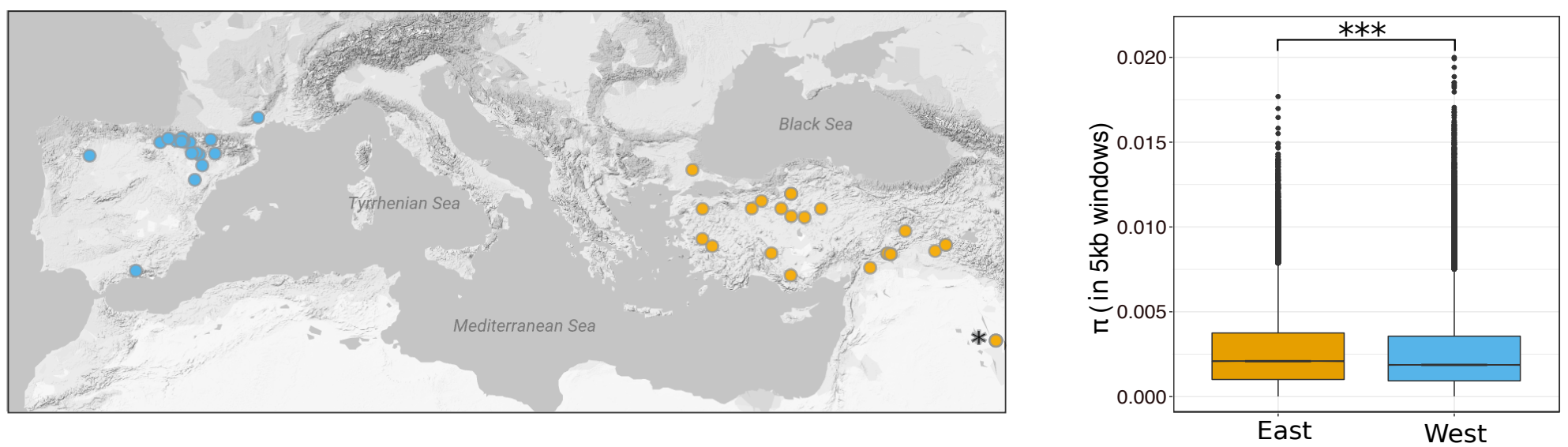

B

D
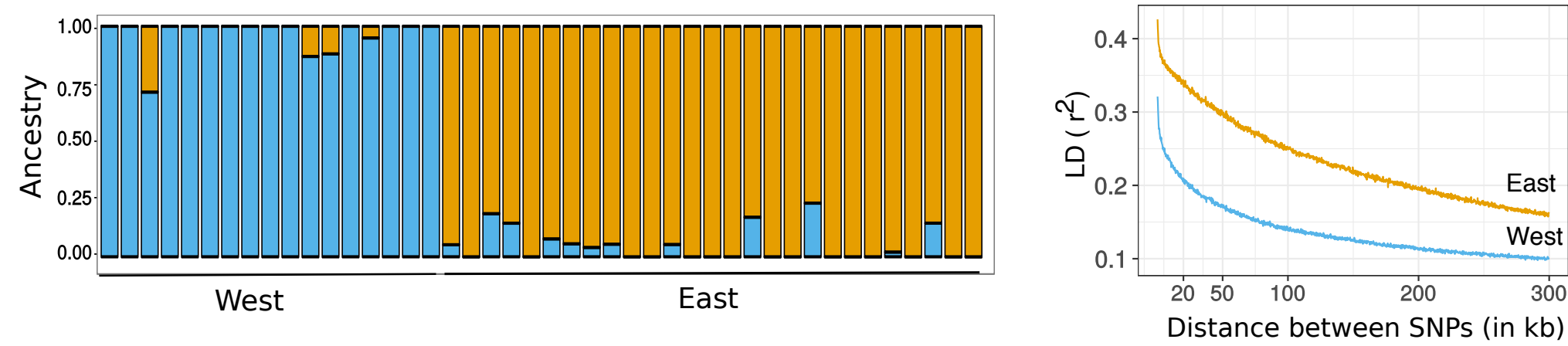


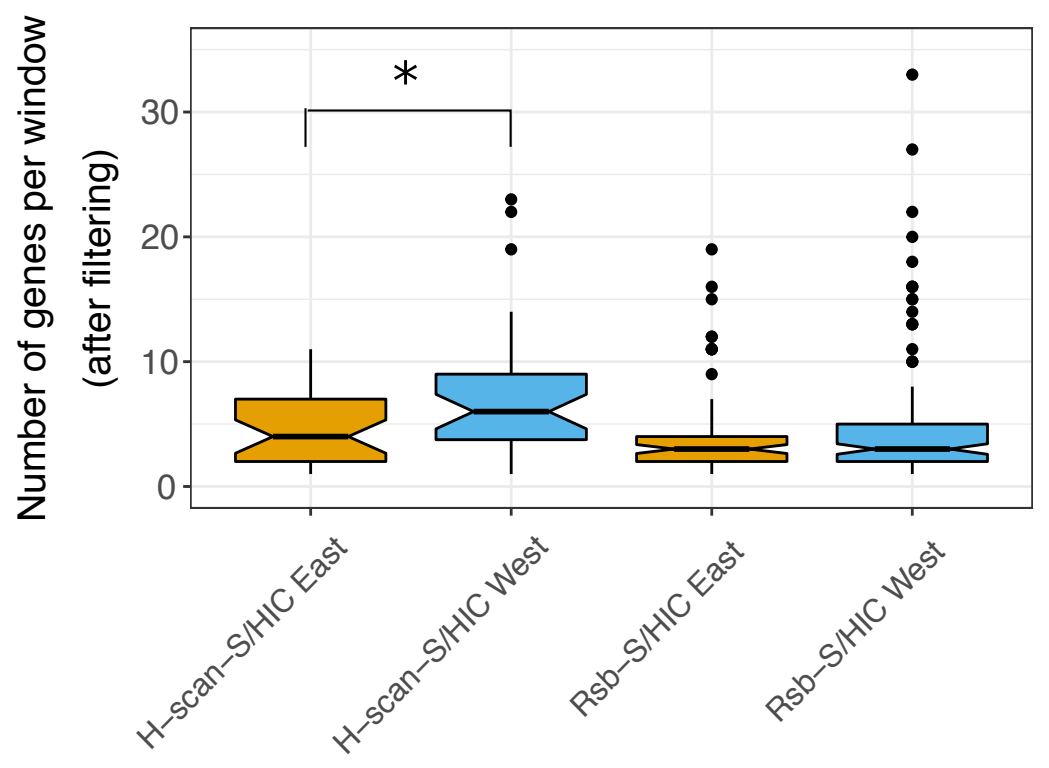

B

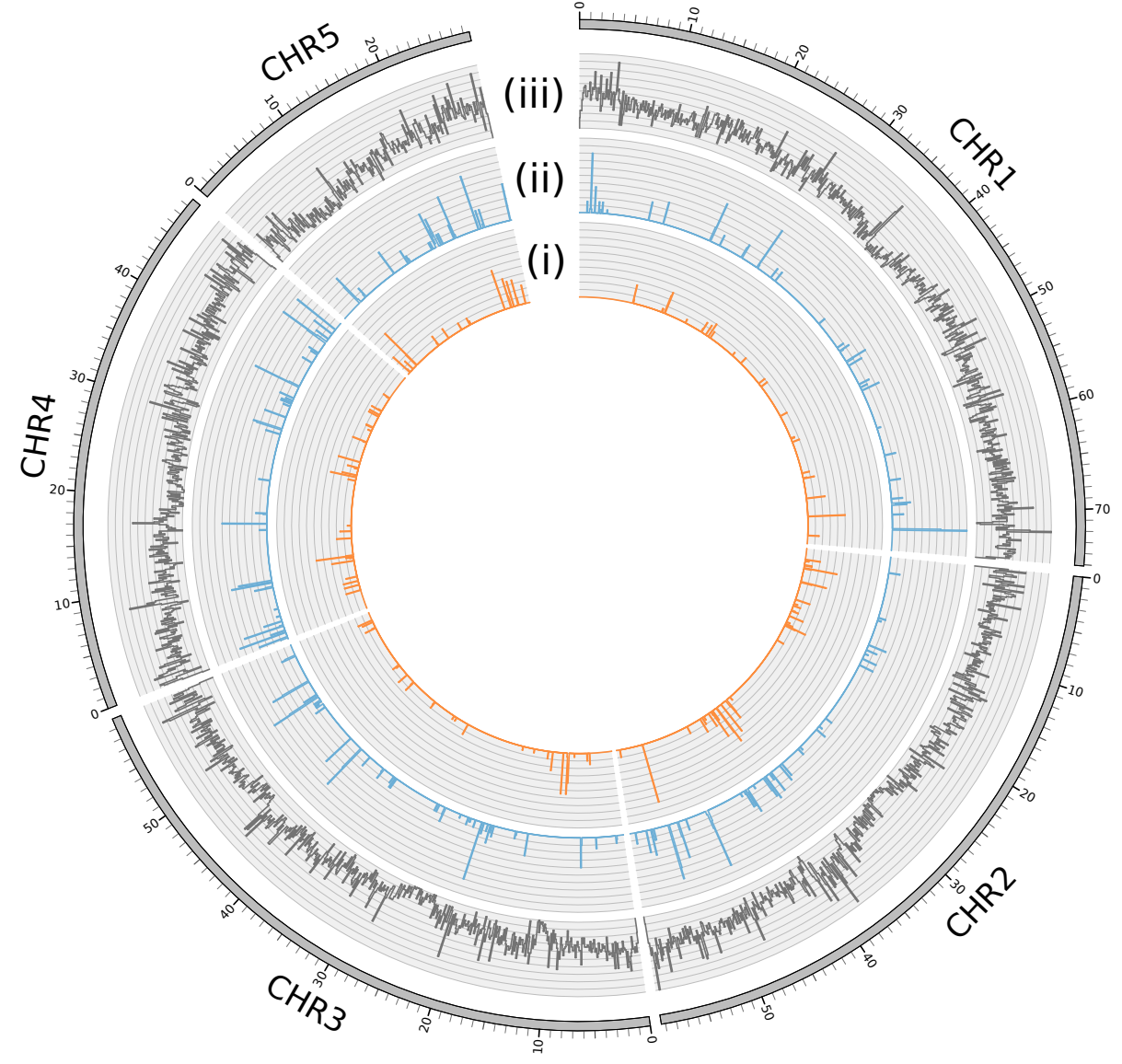



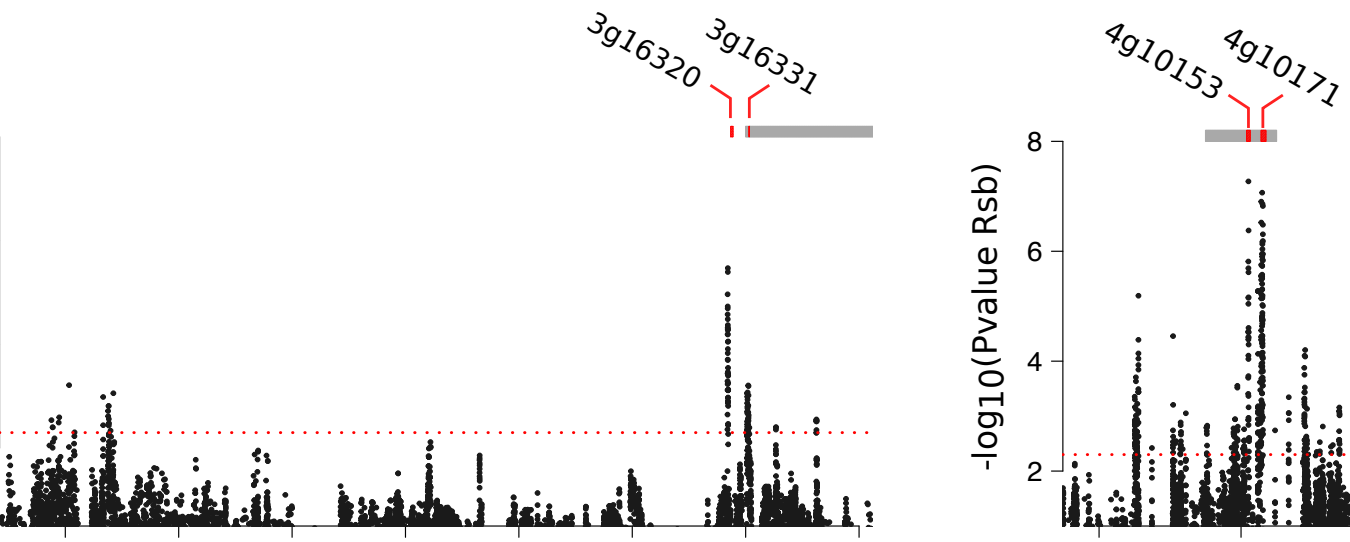

B

West

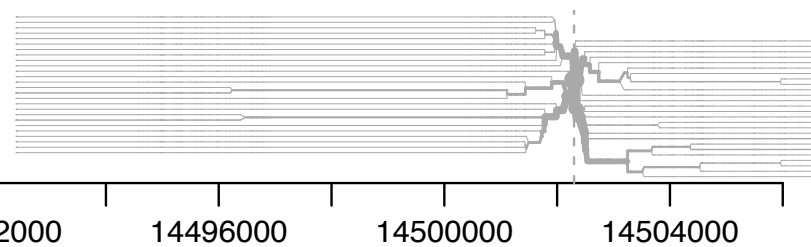

East

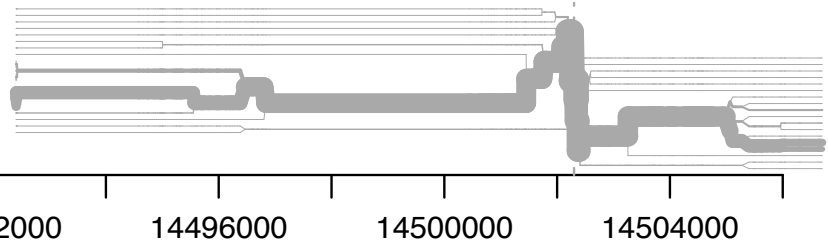

Chromosome 3

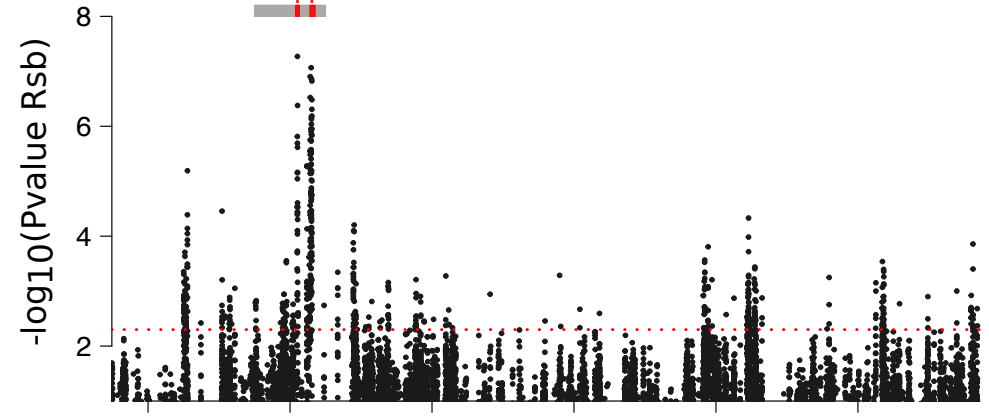

10600000
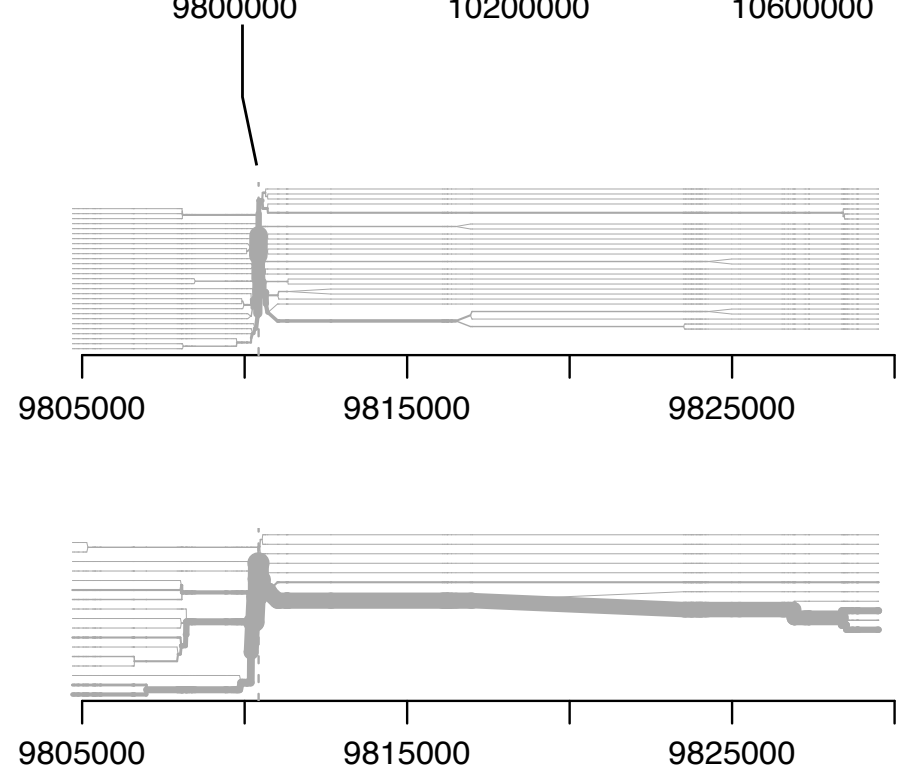

Chromosome 4 